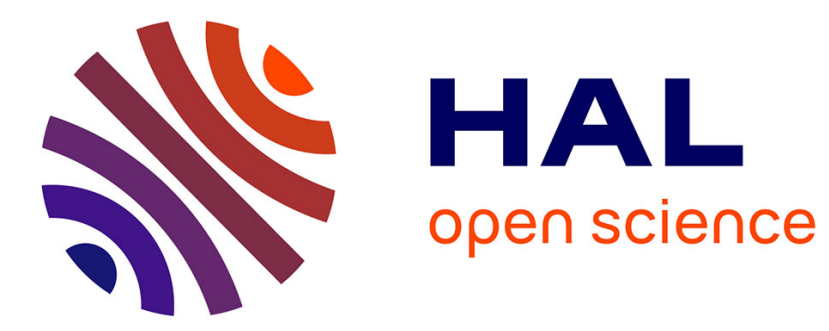

\title{
L'éthique de la recherche en psychiatrie adulte
}

\author{
N. Bouaziz, S. Boussion, R. Benadhira, S. Braha, A. Wojakiewicz, D. Januel
}

\section{To cite this version:}

N. Bouaziz, S. Boussion, R. Benadhira, S. Braha, A. Wojakiewicz, et al.. L'éthique de la recherche en psychiatrie adulte. Annales Médico-Psychologiques, Revue Psychiatrique, 2011, 169 (6), pp.367. 10.1016/j.amp.2010.04.023 . hal-00771588

\section{HAL Id: hal-00771588 https://hal.science/hal-00771588}

Submitted on 9 Jan 2013

HAL is a multi-disciplinary open access archive for the deposit and dissemination of scientific research documents, whether they are published or not. The documents may come from teaching and research institutions in France or abroad, or from public or private research centers.
L'archive ouverte pluridisciplinaire HAL, est destinée au dépôt et à la diffusion de documents scientifiques de niveau recherche, publiés ou non, émanant des établissements d'enseignement et de recherche français ou étrangers, des laboratoires publics ou privés. 


\section{Accepted Manuscript}

Title: L'éthique de la recherche en psychiatrie adulte

Authors: N. Bouaziz, S. Boussion, R. Benadhira, S. Braha, A. Wojakiewicz, D. Januel

PII: $\quad$ S0003-4487(11)00066-7

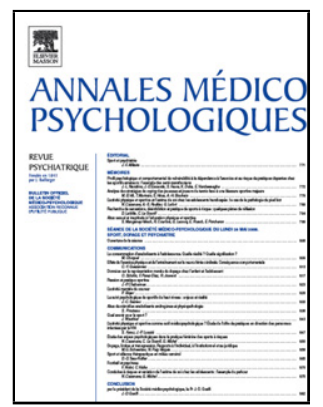

DOI: $\quad$ doi:10.1016/j.amp.2010.04.023

Reference: $\quad$ AMEPSY 1310

To appear in: Annales Médico-Psychologiques

Received date: $\quad 26-2-2010$

Accepted date: $\quad 11-4-2010$

Please cite this article as: Bouaziz N, Boussion S, Benadhira R, Braha S, Wojakiewicz A, Januel D, L'éthique de la recherche en psychiatrie adulte, Annales mediopsychologiques (2010), doi:10.1016/j.amp.2010.04.023

This is a PDF file of an unedited manuscript that has been accepted for publication. As a service to our customers we are providing this early version of the manuscript. The manuscript will undergo copyediting, typesetting, and review of the resulting proof before it is published in its final form. Please note that during the production process errors may be discovered which could affect the content, and all legal disclaimers that apply to the journal pertain. 


\title{
Mémoire
}

\section{L'éthique de la recherche en psychiatrie adulte}

The ethics of research in adult psychiatry

\section{N. Bouaziz, S. Boussion, R. Benadhira, S. Braha, A. Wojakiewicz, D. Januel}

EPS Ville Evrard, 202 avenue Jean Jaurès, 93332 Neuilly sur Marne cedex, France

Auteur correspondant : N. Bouaziz, EPS Ville Evrard, 202 avenue Jean Jaurès, 93332 Neuilly sur Marne cedex, France

Texte reçu le 26 février 2010 ; accepté le 11 avril 2010

\section{Résumé}

La recherche médicale a pour but d'améliorer la condition humaine en développant un corps de connaissances valides et fiables. Cependant, l'histoire de la médecine au cours des siècles fut marquée par de nombreux scandales montrant les dérives possibles de la recherche lorsque celle-ci n'est pas encadrée par une réflexion éthique ni par la loi. La réflexion éthique est donc permanente et les chercheurs doivent sans cesse interroger leur pratique. En psychiatrie, l'objet de la recherche est un être humain rendu vulnérable par ses troubles. Aussi, dans cet article, nous nous proposons d'examiner les fondements théoriques de l'éthique dans la recherche sur l'être humain. Il s'agit donc d'une réflexion sur la signification réelle de ce concept, son histoire, ses principes et ses particularités dans le domaine de la psychiatrie.

Mots clés : Éthique ; Psychiatrie ; Recherche ; Volontaires

\begin{abstract}
Medical research aims to improve the human condition by developing a body of knowledge valid and reliable. The history of medicine over the centuries, however, was marked by numerous scandals showing the possible misuse of research when it is not framed with an ethical, or the law. The ethical debate is ongoing and researchers must continually examine their practices. In psychiatry, the object of research is a human being made vulnerable by his trouble. The aim of this article is examine the theoretical foundations of
\end{abstract}


ethic in research on human being. It is therefore a reflection of the true significance of this concept its history, its principles and particularities in the field of psychiatry.

Keywords: Ethics; Psychiatry; Research; Volunteers

\section{Introduction}

La recherche a pour but d'améliorer la condition humaine en développant un corps de connaissances valides et fiables. Dans son rapport [9], le comité consultatif national d'éthique (CCNE) précise que «chercher à connaître scientifiquement l'être humain est un bien, mais cela ne peut se faire au prix de la justice, de la sécurité ou de l'autonomie de personnes ». Ce propos est illustré par l'histoire de la médecine qui fut marquée par de nombreux scandales montrant les dérives possibles de la recherche lorsque celle-ci n'est pas encadrée par une réflexion éthique ou par la loi. La réflexion éthique doit donc être permanente, et les chercheurs doivent sans cesse interroger leur pratique. En psychiatrie, cette obligation est renforcée par le fait que la recherche s'adresse à des sujets rendus vulnérables par la nature même de leurs troubles.

Dans ce travail, nous nous proposons d'examiner les fondements théoriques de l'éthique dans la recherche sur l'être humain, son histoire, ses principes et ses particularités dans le domaine de la psychiatrie, tout en gardant à l'esprit que les questions posées par l'éthique dans la recherche ne peuvent que rester ouvertes.

\section{Définition de l'éthique}

Le mot éthique provient du latin ethicus, la morale, et du grec ethos, la coutume, les mœurs. Il qualifie les réflexions théoriques portant sur des pratiques et leur condition de réalisation. Cette discipline envisage les questions qui se posent dans la délibération et la prise de décision ; elle tente de répondre à la question : «Que dois-je faire ? Elle possède donc une dimension subjective mais s'attache aussi aux valeurs et se détermine de manière relative dans le temps et dans l'espace, en fonction de la communauté humaine à laquelle elle s'intéresse. Selon D. Sicard, le président du CCNE [10] : «L'éthique ne donne pas de recettes ... J'ai le sentiment que l'éthique est de l'ordre de l'interrogation; de l'inquiétude. » $\mathrm{Ce}$ même auteur précise : «Une réflexion éthique qui à un moment donné serait considérée comme close serait par essence non éthique » [3]. La réflexion éthique suscite le débat, la 
confrontation des idées, elle pousse à la révision des certitudes, c'est une pensée toujours en mouvement. «En tant que système normatif, l'éthique est l'ensemble de principes exprimant des valeurs liées à des exigences morales (faire le bien, éviter de nuire) ou à des choix culturels qui ont une dimension morale [12]. De ces principes découlent des recommandations et des règles s'adressant à un groupe social déterminé, par exemple : les membres d'une profession pour la déontologie » [4].

Il ne peut y avoir de réflexion éthique sans le développement de l'esprit critique. En effet, la question éthique consiste à examiner de façon critique la façon dont l'être humain s'inscrit dans les relations qui le constituent, le sollicitent, au sein desquelles il doit prendre position, se situer et répondre librement de lui-même et de ses actes [1,4]. Ainsi, la réflexion éthique est axée sur le concept de responsabilité. Selon Paul Ricœur [18], l'éthique est une demande qui résulte de multiples situations de décisions impliquant des valeurs : «Je ne veux pas laisser réduire l'Éthique à la moralité du devoir. L'Éthique plonge dans le désir d'accomplissement. Pour moi, c'est le fond ultime du problème moral, au sens large du mot. C'est le souhait de réalisation. » Il explique que la deuxième composante de ce désir de « vivre bien », c'est que l'Autre y est toujours impliqué. L'Autre peut être un proche dans des rapports d'amitié ou d'amour, ou plus lointain, dans un cadre professionnel par exemple. Il précise enfin que l'Éthique ne suffit pas. Le désir de vivre bien doit être soumis à l'obligation morale, où il rencontre des interdictions autant que des devoirs.

Enfin, à un troisième niveau, le devoir lui-même doit passer l'épreuve de la décision sage, prudente, face à des situations concrètes singulières [18].

Le Nouveau Petit Robert, quant à lui, donne une définition philosophique de l'éthique : «Science de la morale, art de diriger la conduite. » De même, M. Siksou rappelle que «l'Éthique est inséparable de la philosophie, et beaucoup la considèrent comme une branche de la philosophie morale ». Cette discipline se décline en trois domaines: la métaéthique, l'éthique appliquée et la morale, qu'il convient de distinguer. La métaéthique, aussi appelée «éthique substantielle », étudie les termes moraux dans leur signification, ainsi que l'épistémologie et la métaphysique des jugements moraux (recherche des origines et du sens des concepts moraux) [3]. C'est une éthique analytique, qui permet à l'éthique un retour réflexif sur elle-même [5,16]. Aujourd'hui, l'éthique tente de répondre à des questions auxquelles sont confrontés les professionnels au quotidien, qui se retrouvent dans leur pratique face à des contradictions, malaises, dilemmes et qui constituent le domaine de la recherche d'éthique appliquée ou éthique pratique. En effet, Feldmann et al. [14] précisent que «l'éthique pratique ne veut pas se perdre dans des argumentations trop abstraites et trop 
poussées, qui contribuent certes à éclairer les problèmes, mais dont on soupçonne qu'elles ne peuvent véritablement aboutir à leurs solutions ». Ainsi, cette éthique appliquée est à visée plus prescriptive que normative, et soucieuse des conséquences de l'action, s'attache à analyser des situations bien précises [7]. Enfin, la morale est un ensemble de règles ou de lois ayant un caractère universel. C'est un héritage commun de principes et normes relatives au bien et au mal qui permettent de qualifier et de juger les actions humaines. Les normes sont intériorisées et rendent compte de ce qu'une personne doit faire, est autorisée à faire, ou a le droit de faire. La morale et l'éthique sont indissociables : l'éthique apparaît lorsque la morale entre en crise, c'est le dépassement de la morale. L'éthique intervient lorsqu'il y a un doute, un conflit. On peut considérer qu'il s'agit d'une réflexion sur les fondements de la morale. Selon M. Siksou, la morale est ce que l'on fait par devoir (de l'ordre de la volonté), alors que l'éthique est ce que l'on fait par amour (de l'ordre du sentiment) [3].

\section{3. Évolution de la réflexion éthique au cours de l'histoire}

De l'Antiquité au $\mathrm{XX}^{\mathrm{e}}$ siècle, de nombreuses expérimentations à visée de recherche ont été pratiquées sur l'homme sans aucune préoccupation éthique. B. Halioua en témoigne dans son ouvrage Science et conscience, Histoire de l'éthique médicale [15]. Il donne pour exemple de nombreuses expériences pratiquées sans le consentement du sujet, oubliant «l'interdit qui frappait les recherches dangereuses et sans bénéfice thérapeutique direct». Pour n'en citer que quelques-unes : le chirurgien du roi Louis XIV, en 1686, s'entraînait sur des patients souffrant du même mal que le souverain et expérimenta ainsi différentes techniques chirurgicales ; Louis Pasteur, en 1884, désirait tester son vaccin contre la rage sur des condamnés à mort...

$\mathrm{Au} \mathrm{XIX}^{\mathrm{e}}$ siècle, la théorie de l'évolution de Charles Darwin ainsi que les travaux de Mendel ont été utilisés à tort par certains pour justifier des théories racistes, tel F. Galton qui «créa » la notion d'eugénisme en 1883. Ce concept - qui s'appuie et interprète certaines notions de la génétique et prétend étudier et mettre en œuvre les moyens d'améliorer l'espèce humaine, en cherchant soit à favoriser l'apparition de certains caractères, soit à éliminer les maladies héréditaires - a été à l'origine de graves dérives au cours du $\mathrm{Xx}^{\mathrm{e}}$ siècle. À la fin de la Seconde Guerre mondiale, le procès de Nuremberg intenté contre 24 des principaux responsables du régime nazi accusés de complot, crime contre la paix, crimes de guerre et crimes contre l'humanité, est un moment clé dans le questionnement éthique. Lors de ce procès ont été jugés les médecins nazis qui avaient pratiqué des expérimentations médicales 
sur les prisonniers des camps de concentration. Pour leur défense, les accusés ont mis en avant l'absence de cadre et de législation autour de la question des expérimentations humaines. Ce procès a permis d'attirer l'attention sur les dérives possibles commises au nom de la science. Les quatre juges américains estimèrent donc indispensable d'élaborer un code de droit international permettant de qualifier les bonnes pratiques de la recherche menée sur l'homme, ce qui mena à la création du Code de Nuremberg. Ce code propose 12 règles, posant les principes en matière d'expérimentation médicale sur des sujets humains. Il en ressort l'obligation d'obtenir le consentement volontaire du sujet humain et la nécessité d'éviter toute souffrance et/ou dommage physique et mental. Enfin, les risques encourus ne devront jamais excéder l'importance humanitaire du problème que doit résoudre l'expérience envisagée.

La réflexion ayant poussé à la création du Code de Nuremberg sera poursuivie et approfondie, un an plus tard, dans la Déclaration universelle des droits de l'homme, puis par la suite dans la Déclaration d'Helsinki, adoptée par l'AMM (Association Médicale Mondiale) en 1964. Depuis, celle-ci a été amendée six fois, la dernière datant de l'Assemblée générale d'octobre 2008. Il s'agit d'une déclaration de principes éthiques qui a pour objectif de fournir des recommandations aux médecins et autres participants à la recherche médicale sur des êtres humains. Ce document insiste sur le devoir du médecin de «promouvoir et sauvegarder la santé des personnes impliquées dans la recherche» (art. 3). Il précise que « le bien-être de chaque personne impliquée doit prévaloir sur tous les autres intérêts » [15].

En Amérique du Nord, un cadre éthique pour la recherche s'est développé bien après l'Europe, dans les années 1960. En effet, selon H. Doucet, « la science américaine ne se sentit nullement concernée par les normes morales édictées par les juges du tribunal de Nuremberg » [13]. Il explique cela par le fait que les États-Unis venaient de remporter la guerre, et un grand optimisme régnait à l'époque sur la science. Les chercheurs et médecins américains pensaient à tort que ces expériences cruelles avaient été conduites non pas par des médecins, mais par des fonctionnaires nazis sadiques: par conséquent, les véritables chercheurs n'avaient rien à apprendre de cette expérience. Or, en 1966, Henry Beecher mobilise l'opinion publique en publiant dans le New England Journal of Medicine un article intitulé «Ethics and Medical Research» [2]. Il y décrit vingt-deux expériences qu'il qualifie de non éthiques, comme par exemple injecter des cellules cancéreuses vivantes à des personnes âgées et séniles placées en institution pour éprouver leur résistance immunologique au cancer : «Ces expérimentations n'étaient pas sans danger pour les individus utilisés, ne leur apportaient aucun bénéfice et ignoraient la nécessité du consentement. » Mais il explique 
que la majorité des médecins chercheurs ne prenaient guère au sérieux les critiques qui leur étaient adressées, "l'intérêt de faire des progrès au bénéfice des malades de demain passait avant le respect à l'égard des sujets d'expérimentation » [13]. Suite à cela, de nombreuses expériences non éthiques, menées entre autres par l'armée ou encore par le Département américain de l'Énergie, ont indigné le public. Il y eut une prise de conscience collective, et les NIH (National Institute of Health) durent agir : la crainte de nuire à la recherche les avait jusqu'alors empêchés d'agir et d'imposer des normes. Ils mirent en place des IRB, Institutional Review Board, comités locaux composés de chercheurs et de profanes appelés à évaluer des projets de recherche. Ils se sont imposés dans tous les milieux de la recherche, aussi bien aux États-Unis qu'en Europe.

Dès 1968, le Congrès s'intéressa à l'éthique : le premier projet de loi fut présenté par le sénateur Mondale. Il suscita la colère des médecins : le congrès devait, selon eux, se limiter à financer la recherche et faire confiance aux experts pour le reste. En 1973, la première commission nationale pour la protection des sujets humains en recherche médicale fut établie (elle était limitée à la recherche et n'avait qu'un pouvoir de recommandation). Son retentissement fut considérable, puis elle publia le rapport Belmont, qui «présentait les principes éthiques de base qui devaient guider toute recherche sur les sujets humains » (The National Commission, 1982) [13].

\section{Structures et législation consacrées à l'éthique en France}

Créé en 1983, le CCNE est le premier des comités d'éthiques nationaux au monde. C'est une instance nationale, composée d'une quarantaine de membres. Selon la loi du 6 août 2004, « Le Comité Consultatif National d’Éthique pour les sciences de la vie et de la santé a pour mission de donner des avis sur les problèmes éthiques et les questions de société soulevées par les progrès de la connaissance dans les domaines de la biologie, de la médecine et de la santé ». Sa mission première est donc de produire des avis et rapports sur les questions dont il est saisi. Il n'est doté d'aucun pouvoir normatif ni prescriptif, mais seulement d'un pouvoir consultatif, et ne rend pas d'avis sur des situations particulières. $\mathrm{Ce}$ comité agit à un niveau transversal, sans interférence directe avec les situations de santé sur le terrain. En 1995, un nouveau type de structure est mis en place : l'Espace éthique, ouvert à tous. Ce modèle s'oppose à celui de comité, qui se définit par la notion d'appartenance. C'est donc un lieu d'échange, de recherche et de délibérations ouvert à tous les partenaires impliqués dans les situations de santé, avec pour objectif de promouvoir la réflexion éthique 
sur les pratiques de la santé et des soins. Depuis une dizaine d'années, les organismes de recherche français eux-mêmes (Inserm - Institut national de la santé et de la recherche médicale, INRA - Institut national de recherche agronomique...), se dotent de comités d'éthique. «Cette émergence de dispositifs et d'initiatives sur l'éthique doit toujours appeler un regard critique, particulièrement s'agissant des comités. En effet, tout dispositif qui prétend posséder une légitimité dans les champs de l'éthique, supérieure à celle que l'on peut avoir de façon générale dans la réflexion que l'on porte soi-même sur la signification et les questions que suscitent ses propres activités professionnelles, doit nous interroger : d'où viendrait en effet une telle légitimité ? Nombre de comités récemment créés sont autoproclamés et prétendent à une autorité dans le domaine » [3].

La loi Huriet Sérusclat, loi de protection des personnes se prêtant à la recherche biomédicale, créée le 20 décembre 1988, impose un cadre juridique à la recherche. À l'origine, elle fut conçue pour encadrer les expériences pharmaceutiques, puis a été étendue à toute expérimentation sur l'homme. Consolidée en 2000, elle affirme la légalité de la recherche et se compose de quatre points principaux : la nécessité d'une logique de protection des personnes dans la recherche biomédicale (cette loi différencie les recherches biomédicales avec ou sans bénéfice individuel direct), la définition claire des statuts de promoteur et d'investigateur, l'obligation d'un consentement libre, éclairé et exprès, et la mise en place de CCPPRB : on ne peut conduire la recherche biomédicale qu'après soumission du protocole appliqué à ce comité indépendant et spécifique. Il sera renommé CPP (Comité de Protection des Personnes) en 2004. Certains éléments de cette loi sont contestés par Caverni [8], notamment le fait que le chercheur doive travailler avec un médecin, seul habilité à saisir le CPP. Or, en psychologie, en physiologie, génétique et neurosciences, les chercheurs ne sont pas médecins. La loi a éclairé le motif de la présence du médecin : «La loi qualifie d'investigateur, et non pas de directeur de recherche, le médecin qui dirige et surveille la recherche au regard des exigences tenant à la protection des personnes. » Seul le médecin sera l'interlocuteur du CPP, il est en mesure d'apprécier les conséquences de la recherche pour la santé des personnes qui s'y prêtent. Cependant, la recherche comportementale menace rarement la santé des sujets. Il faudrait qu'il puisse être saisi par un chercheur non-médecin.

Enfin, la loi du $1^{\mathrm{er}}$ juillet 1994 sur le «traitement des données nominatives à des fins de recherche » constitue une dérogation au secret professionnel. Avant 1994, réaliser une recherche comportant le traitement informatique de données nominatives posait problème par rapport au secret. Cette loi affirme que «toute personne a le droit de s'opposer à ce que des données nominatives le concernant fassent l'objet d'un traitement automatisé ». Il y a deux 
étapes : le CCTIR (Comité Consultatif sur le Traitement de l'Information en matière de Recherche dans le domaine de la santé) émet un avis, et la CNIL (Commission Nationale Informatique et Liberté) donne une autorisation.

Dans de nombreux pays scientifiquement évolués, ont été mis en place des codes de conduite à respecter concernant les questions éthiques de la recherche comportementale (différents de ceux de la recherche médicale), ainsi que des instances spécifiques prises en charge par les chercheurs et les institutions de recherche. Caverni [8] déplore le fait que ce ne soit pas le cas en France, où il n'existe aucun code concernant spécifiquement la recherche comportementale. Pour pallier cela, il propose un code de conduite des chercheurs dans les sciences du comportement humain et les modalités de sa mise en œuvre, adopté par la société française de psychologie (SFP). L'objectif était de poser les jalons pour la constitution d'un comité d'éthique en proposant une charte consensuellement acceptée par la communauté scientifique. Cette charte a été inspirée des codes des deux plus importantes sociétés savantes de la discipline : l'APA (American Psychological Association) et la BPS (British Psychological Association).

\section{Principes fondamentaux de l'éthique du chercheur}

Ces principes sont illustrés par le rapport Belmont, une réflexion conduite aux ÉtatsUnis sur l'éthique en 1979. Il a mené à trois grands principes que tout chercheur doit respecter : autonomie, bienfaisance et non malfaisance, justice. Plus tard, en 1993, le CCNE rappelle que «toute investigation expérimentale sur l'être humain doit se faire selon une démarche scientifique irréprochable, dans le respect de la liberté d'action des personnes, de leur sécurité, du principe de justice; et que le consentement libre, éclairé et exprès des personnes qui se prêtent à la recherche ne décharge pas les chercheurs de leur responsabilité morale et scientifique ». Il rappelle qu'une étude sur l'être humain « ne doit pas être l'occasion de manipulation ni de discrimination, et que la chaîne du secret professionnel doit être sans faille ». Nous allons revoir en détail ces principes fondamentaux.

\subsection{Principe de respect et d'autonomie des personnes}

L'autonomie se définit comme la capacité d'agir de façon responsable à son propre égard et à l'égard des autres. De la volonté de respecter chacun dans son autonomie découle automatiquement le principe du consentement aux soins. Selon le British Psychological 
Association (BPS) et l'American Psychological Association (APA), le principe est que «normalement» et «autant que possible» le chercheur doit obtenir le «consentement valide » et «éclairé » des personnes qui se prêtent à la recherche. La personne doit savoir qu'elle possède la liberté d'y participer ou non. Au préalable, la personne doit être informée de tous les aspects susceptibles d'influencer son consentement (risques, inconfort, effets négatifs).

L'obtention du consentement éclairé peut poser quelques problèmes, comme certaines recherches en psychologie impossibles à mener si les buts de la recherche sont dévoilés. Le volontaire n'est donc pas totalement informé des objectifs de la recherche car cela pourrait modifier et/ou influencer son comportement, représentant ainsi un biais. Dans ce cas, le chercheur donnera une information complète à la fin de la recherche. Le CCNE, quant à lui, prend en compte cette notion dans l'avis $\mathrm{n}^{\circ} 38:$ : Si certains aspects de la recherche sont délibérément cachés dans l'intérêt de l'étude, les sujets recevront à la fin de la recherche des explications complètes sur les objectifs de la recherche et l'usage qui va en être fait. À ce moment, la personne pourra infirmer ou confirmer son consentement. » Le sujet pourra toujours exiger que les informations le concernant lui soient remises ou détruites. Caverni [8] considère que, si des éléments de tromperie sont apportés, ils doivent toujours avoir une justification scientifique forte, et il faut s'assurer que c'est la seule alternative. Selon lui, la loi est parfois excessive : on ne peut pas donner aux personnes toutes les informations sur la recherche, et il n'est pas indispensable d'avoir systématiquement un consentement exprès, qui lève l'anonymat.

\subsection{Principe de bienfaisance et de non-malfaisance}

Ces deux notions se complètent. Leur articulation conduit à une réflexion sur la proportionnalité des actions encourues dans le soin d'une personne : les risques encourus sont-ils justifiés par les bénéfices attendus d'une telle intervention? Le chercheur doit vérifier que la balance bénéfices/risques est positive : cela signifie qu'il faut tenter de maximiser les bénéfices escomptés, et de minimiser les inconvénients possibles. Il doit toujours se rappeler que la recherche sur l'être humain doit se fonder sur l'état des connaissances scientifiques actuelles et doit avoir pour but d'accroître les connaissances de l'être humain. Ce principe implique de poser trois questions avant d'envisager une action :

- La finalité de l'action entreprise correspond-elle à un intérêt pour la personne concernée ? 
- Est-il possible que cette action soit nuisible à la personne ?

- Le rapport risques/bénéfices permet-il de considérer cette action comme justifiable ?

En effet, le risque peut être mineur, c'est-à-dire du même ordre que ceux encourus dans la vie quotidienne, mais il peut aussi être sérieux, ce qui nécessite un contrôle élevé avant, pendant, et après l'expérimentation.

\subsection{Principe de justice}

Les charges de la recherche doivent être équitablement réparties: les sujets de recherche ne doivent pas être puisés toujours dans la même population en raison de dérives, qui, dans le passé, ont conduit à s'adresser à des populations plus vulnérables. Ainsi, ceux qui supportent la recherche doivent être aussi ceux qui bénéficient de ses résultats. Le principe de justice avance les notions d'intégrité, de dignité, de vulnérabilité. La notion d'intégrité renvoie aux dimensions physiques et psychiques de la personne, dans une dimension culturelle et historique personnelle. La prise en compte de l'intégrité des personnes impose une reconnaissance et un respect de ce qu'elles sont à titre individuel. La vulnérabilité est la capacité à être blessé, ou atteint. C'est une composante importante de la réflexion sur l'éthique des pratiques. Enfin, la dignité de la science et de ses exigences méthodologiques signifie honnêteté, rigueur et compétence dans la recherche (CCNE).

\section{Particularités de la recherche avec des sujets souffrant de troubles mentaux}

Lors d'une recherche menée avec des sujets souffrant de troubles psychiques, il est indispensable de prendre en compte les conséquences du trouble mental sur le libre arbitre du patient. Ainsi, A. Danion-Grilliat [11] insiste sur le fait que «le sujet idéal de l'éthique est bousculé par la maladie mentale », c'est-à-dire que la maladie mentale remet en question l'information et l'autonomie du sujet se prêtant à la recherche. Sa vulnérabilité représente un impératif éthique, d'autant plus qu'elle peut se trouver majorée par des difficultés de compréhension et de communication. Cependant, les difficultés de compréhension des sujets schizophrènes, par exemple, ne sont pas les mêmes en fonction de l'évolution de la maladie. Pour informer, il faut tenir compte de la capacité de compréhension du patient. L'information peut être «parasitée» par son activité fantasmatique. De plus, le trouble peut affecter les 
capacités cognitives, ou peut relever d'une activité délirante qui déforme l'information, voire la dévie. L'information doit donc être adaptée au patient.

Le Code de Nuremberg prévoit le principe de protection des personnes pour celles qui ne peuvent donner un tel consentement. Le chercheur doit obtenir une autorisation appropriée d'une personne légalement autorisée. Pour les mineurs, il doit être donné par le titulaire ou détenteur de l'autorité parentale, le représentant légal ou le juge des tutelles, mais il faut tout de même avoir le consentement du mineur. Selon Bourguignon, ces sujets doivent être protégés à proportion de leur incapacité et de leur dépendance.

Le cas le plus complexe est probablement l'obtention du consentement des patients présentant une schizophrénie, pathologie altérant potentiellement les perceptions et les aptitudes de jugement de la personne souffrante. Cependant, des études ont démontré que contrairement aux idées reçues, la capacité au libre arbitre n'est pas altérée dans l'ensemble de cette population de malade. II existe en effet des sous-groupes de patients aptes à donner leur consentement, après un travail d'information et d'explication de qualité [6]. Cette aptitude serait inversement corrélée à l'importance de la psychopathologie, surtout les dimensions négatives, cognitives de la maladie et aussi à la qualité de l'insight [6]. Ainsi, même en phase aiguë de la maladie, ce sous-groupe de patients schizophrènes serait apte à donner un consentement valable au point de vue juridique. Il est donc obligatoire de prendre en compte, pour chaque sujet, la sévérité de sa maladie et son interférence avec l'aptitude à avoir un jugement fiable pour donner un consentement. Les auteurs soulignent l'intérêt de mettre en place des études évaluant, au cours des différents stades de la maladie, la capacité de consentir et aussi l'éventuel impact positif des thérapies (cognitives et comportementales) sur l'insight et la capacité à fournir un consentement [6,17].

\section{Démarche éthique dans l'élaboration d'un protocole}

Selon le CCNE, tout projet de recherche scientifiquement invalide ou inutilement redondant doit être éliminé : «Le sujet humain ne saurait être utilisé que dans la mesure où sa participation entraîne la probabilité d'un résultat appréciable pour le bien commun, fût-ce une avancée de la connaissance de l'homme. » Toute recherche engage la responsabilité du chercheur. La recherche mise en place doit impérativement appartenir à son domaine de compétences, ce qui lui permet d'évaluer sa faisabilité et d'éviter des biais. Les chercheurs ont pour obligation de maintenir et développer leurs compétences professionnelles, et 
reconnaître leurs limites. Ils doivent rendre leurs travaux publics, et veiller à leur respect afin d'éviter tout mésusage des résultats de leur recherche. Le chercheur peut être tiraillé entre l'intérêt scientifique visé et l'efficacité de la méthodologie, et les coûts financiers et humains (risques, inconvénients, astreintes). Il doit être vigilant face à de possibles déviances, dont la fuite d'informations.

Les paramètres à prendre en compte sont tout d'abord le choix du thème, de l'hypothèse de la recherche, ainsi que ses objectifs. Dans l'idéal éthique : «Le chercheur est pleinement responsable de ce qu'il fait, veille à respecter la dignité des personnes, de luimême et de la science, et donc ne propose et ne réalise que des projets éthiquement corrects » [3].

L'hypothèse ne doit pas être accusatrice ou dévalorisante, mais doit respecter les personnes concernées. Les critères d'inclusion doivent reposer sur des aspects éthiques. Il faut présenter la recherche aux personnes retenues en restant clair et expliquer clairement les objectifs de la recherche. Le chercheur ne doit pas oublier, selon le même auteur, que c'est lui qui est en position de demandeur, et il doit donc établir avec le sujet un lien de partenariat.

L'environnement doit assurer anonymat et confidentialité. Il est nécessaire d'utiliser des outils valides et appropriés à la recherche. Lors du recueil des données, il faut assurer le bien-être du sujet, aussi bien physique que mental. Dans l'exploitation des données, le chercheur doit avoir le souci d'être le plus objectif et honnête possible, par exemple en présentant également les résultats qui ne concordent pas avec l'hypothèse de départ. Enfin, le chercheur doit prendre conscience de l'impact que l'intervention peut avoir sur le sujet, et penser aux conséquences de la recherche. Le risque est d'être obnubilé par l'objectif final et de ne pas voir les effets de la recherche sur le sujet. «Le chercheur est pleinement responsable de ce qu'il fait, veille à respecter la dignité des personnes, de lui-même et de la science, et donc ne propose et ne réalise que des projets éthiquement corrects » [3]. C'est pourquoi, en France, toute recherche conduite sur des sujets humains doit nécessairement obtenir l'avis d'un Comité de Protection des Personnes.

\section{Responsabilité du chercheur et lien avec le sujet se prêtant à la recherche}

D’après Paul Ricœur [18], « le respect des personnes implique l'estime de soi, la façon dont on traite les autres révélant la façon dont on se traite soi-même ». Le chercheur est un individu avec son histoire, sa culture, sa sensibilité, ses pensées qui vont composer l'ensemble de sa personnalité et être à l'origine de ses comportements, allant influencer sa recherche. 
Pour G. Devereux (1980), « une science réaliste de l'humanité ne peut être créée que par les hommes qui sont les plus conscients de leur propre humanité ». Il explique que le chercheur est soumis à des pressions culturelles latentes qui guident ou abusent la pensée, et les nomme des «déformations contre-transférentielles », qu'il tentera sans cesse de diminuer ou neutraliser. Il est conseillé de «prendre conscience de ses propres réactions et contrôler ses propres manifestations émotionnelles » [3]. O. Bourguignon insiste sur ce point en précisant que pour certains psychologues, «s'interroger sur les valeurs engagées dans l'action est vécue comme une mise en question de soi ». Ainsi, elle estime que certains chercheurs en sciences humaines ont une attitude défensive vis-à-vis des questions éthiques. Selon elle, cette « réticence » viendrait du fait que ces chercheurs considèrent que ces dispositions vont à l'encontre de leur autonomie. Elle explique qu'ils « redoutent l'incompétence des évaluateurs en ce qui concerne leur propre projet ou les rapports de pouvoir existant au sein des comités d'évaluation » [3].

Il est indispensable que le chercheur en psychologie prenne en compte les conséquences de sa recherche et la façon dont il va obtenir ses résultats. Il doit vérifier l'impact de l'intervention sur le sujet. Dans leur article «Position du chercheur et souffrance du sujet », Capdevielle et al. [7] tentent d'aborder ce sujet. Pour parvenir à concilier recherche et prise en compte de la souffrance du sujet, ces auteurs proposent de s'orienter vers la psychanalyse qui «offre des repères importants pour penser la pratique ». Leur étude montre que la recherche a pu permettre à certains patients d'avoir un espace de parole où ils pouvaient s'exprimer en toute liberté et être entendus. "L'espace de parole ouvert à l'occasion de la recherche se révèle rapidement être le support de ce "temps pour comprendre" indispensable au sujet pour vivre ce qui lui arrive » [7]. Lorsque le chercheur a une demande vis-à-vis du patient, cela va provoquer une attente de celui-ci. « Le sujet est alors compromis par sa participation à la recherche. Il en sort inévitablement changé : il y a pour lui un avant et un après la recherche. »

Kestemberg [in 3] parle de «bénéfices psychiques » obtenus dans le cadre de la recherche, et c'est pourquoi le chercheur peut être amené à orienter le sujet vers un collègue pour un travail thérapeutique. Selon Giami et Salamin-Amboise [in 3], il ne faut pas confondre recherche et pratique clinique, et ces auteurs recommandent de distinguer ces deux activités. À ce propos, O. Bourguignon [3] insiste sur le fait qu'«on ne peut mener simultanément recherche et thérapie auprès d'un même sujet », et insiste sur la nécessité de distinguer le «lien de clinique » $\mathrm{du}$ «lien de recherche». En revanche, pour Renault d'Allones (1989), la frontière est fine, et «il est illusoire de distinguer entre situation 
thérapeutique et situation de recherche » [3]. Capdevielle et al. [7] vont en ce sens en précisant qu'il n'y a pas d'incompatibilité stricte entre les deux, mais insistent sur la nécessité pour le thérapeute de ne pas utiliser le lien thérapeutique noué avec son patient pour l'amener à participer à une activité de recherche.

\section{Conclusion}

L'éthique est une discipline en évolution permanente dont le questionnement n'est jamais clos. Elle pousse à une perpétuelle remise en question de nos actes, mais aussi de nos valeurs et exigences morales. Il est indispensable que le chercheur mène une telle réflexion, en respectant les principes de bienfaisance, de non-malfaisance, d'autonomie, et de justice. Il résulte de cette réflexion trois exigences pour le chercheur : assurer la liberté des sujets et leur autodétermination en prenant soin de ne pas tomber dans la manipulation, prévenir les dommages et la souffrance, et respecter la vie privée des sujets et de leur famille. Dans la recherche en psychiatrie, ces exigences sont encore plus impératives que la population spécifique à laquelle elle s'adresse est rendue encore plus vulnérable par la nature même des troubles dont elle souffre.

\section{Conflit d'intérêt : à compléter par l'auteur}

\section{Références}

[1] Baudouin N, Bourguignon O. La déontologie des psychologues. L'orientation scolaire et professionnelle.[En ligne], 35/1 | 2006. Mis en ligne le 28 septembre 2009.

[2] Beecher HK. Ethics and clinical research. New England Journal of Medicine 1966;274:1354-6.

[3] Bourguignon O. Éthique et pratique psychologique. Liège: Mardaga; 2008.

[4] Bourguignon O. Questions éthiques en psychologie. Paris: Mardaga; 2003.

[5] Canto-Sperber M. Dictionnaire d'éthique et de philosophie morale. Paris: PUF; 2001.

[6] Capdevielle D, Raffard S, Bayard S, Garcia F, Baciu O, Bouzigues I, Boulenger JP. Competence to consent and insight in schizophrenia: is there an association? A pilot study. Schizophr Res 2009;108:272-9.

[7] Capdevielle V, Laterrasse C, Doucet C. Position de chercheur et souffrance du sujet. Psychologie clinique 1998;5:89-117. 
[8] Caverni JP. Pour un code de conduite des chercheurs dans les sciences du comportement humain. L'année psychologique 1998;98:83-7.

[9] Comité Consultatif National d'Éthique pour les sciences de la vie et de la santé. Avis sur l'éthique de la recherche dans les sciences du comportement humain. Rapport. №38 du 14 octobre 1993. Avaible from: http://www.ccne-ethique.fr/docs/fr/avis038.pdf.

[10] Comité consultatif national d'éthique pour les sciences de la vie et de la santé. Éthique et recherche biomédicale: rapport 1999. Avaible from: http://lesrapports.ladocumentationfrancaise.fr/BRP/014000678/0000. pdf.

[11] Danion-Grilliat A. Le questionnement éthique en psychiatrie (Item 48). Faculté de médecine de strasbourg - module 3b- maturation et vulnérabilité. Année 2006/2007. Avaiblefrom:http://udsmed.ustrasbg.fr/emed/courses/MODULE03B/document/Ethique_psych iatrie.pdf?cidReq=MODULE03B.

[12] Déclaration universelle des droits de l'homme. Avaible from: http://www.un.org/fr/documents/udhr/index.shtml.

[13] Doucet H. Développement des concepts et des enjeux en éthique de la recherche. Pistes 2001;3:1-11.

[14] Feldman J, Canter Kohn R. L'éthique dans la pratique des sciences humaines : dilemmes, ouverture philosophique. Paris: L’Harmattan; 2000. p. 201-21.

[15] Halioua B. Le procès des médecins de Nuremberg : l'irruption de l'éthique médicale moderne. Paris: Vuibert; 2007.

[16] Halioua B. Science et conscience : une histoire de l'éthique médicale. Paris: Liana Levi; 2004.

[17] Jeste DV, Palmer BW, Appelbaum PS, Golshan S, Glorioso D, Dunn LB, Kim K, Meeks T, Kraemer HC. A new brief instrument for assessing decisional capacity for clinical research. Arch Gen Psychiatry 2007;64:966-74.

[18] Ricœur P. Finitude et culpabilité. Paris: Aubier; 2001. 